\title{
Afghan Hound
}

National Cancer Institute

\section{Source}

National Cancer Institute. Afghan Hound. NCI Thesaurus. Code C53911.

The Afghan Hound is a sight hound that is tall and slender with a long, narrow, refined head; and powerful jaws. The occiput is prominent. The long coat is usually the color of sand with a darker face and ear fringes. Height: 27 to 29 inches $(68.58-73.66 \mathrm{~cm}$.)

Weight: 58-64 pounds (26-34 kg.) 